\title{
Evaluation of the Acute Effects of Traditional Persian Medicine Based Hot and Cold Foods on Metabolic Responses and Arterial Stiffness in Healthy Men: Study Protocol for a Randomized Controlled Trial
}

\section{Mohsen Zakerian}

Mashhad University of Medical Sciences

\section{Fatemeh Roudi}

Mashhad University of Medical Sciences

\section{Reza Rezvani}

Mashhad University of Medical Sciences

Malihe Motavasselian ( $\nabla$ MotavasselianM@mums.ac.ir)

Mashhad University of Medical Sciences https://orcid.org/0000-0003-4543-8252

\section{Study protocol}

Keywords: Arterial Stiffness, Metabolic Responses, Temperament

Posted Date: December 22nd, 2020

DOI: https://doi.org/10.21203/rs.3.rs-51623/v1

License: (9) This work is licensed under a Creative Commons Attribution 4.0 International License. Read Full License 


\section{Abstract}

Background: Traditional Persian Medicine (TPM)-based temperament is affected by some determinant factors that seems to have close similarities to resting energy expenditure (REE) and thermic effect of

food (TEF) determinants. Pulse characteristics is considered as a main tool distinguishing different temperaments and vascular function and the probability of cardiovascular events are different in hot and cold-temper individuals in TPM point of view. Additionally, all foods are divided into cold/hot-temper and dry/wet-temper in this medical school and may lead to different responses in subjects regarding their temperaments.

Methods: This experiment will be an acute phase study that will be performed on two groups of healthy individuals with normal body mass index (BMI) with hot and cold temperament. Each person will receive two TPM-based hot/cold isocaloric breakfast meals in 2 different days and metabolic responses and arterial stiffness indices thorough blood samples, indirect calorimetry, and pulse wave analysis and velocimetry will be assessed at fasting state, $0.5,2$, and 4 hours following meal consumption.

Discussion: We will investigate the metabolic differences between individuals with TPM-based hot and cold temperaments and also to evaluate the effects of different TPM-based temperament of isocaloric meals (hot and cold) on metabolic responses and arterial stiffness indices in TPM-based hot/cold temper healthy men in this study. The results of this research project may lead to design of novel combined TPM and modern nutrition-based diet planning to improve metabolic responses and vascular reactivity.

Trial registration: International Clinical Trials Registry Platform IRCT20200417047105N1, retrospectively registered at 19/04/2020. URL: https://irct.ir/trial/47267.

\section{Administrative Information}

Note: the numbers in curly brackets in this protocol refer to SPIRIT checklist item numbers. The order of the items has been modified to group similar items (see http://www.equatornetwork.org/reporting-guidelines/spirit-2013-statement-defining-standard-protocol-items-forclinical-trials/). 


\begin{tabular}{|c|c|}
\hline Title $\{1\}$ & $\begin{array}{l}\text { Evaluation of the acute effect of Traditional Persian medicine based } \\
\text { hot and cold foods on Metabolic Responses and Arterial Stiffness in } \\
\text { healthy men: a cross-over clinical trial }\end{array}$ \\
\hline $\begin{array}{l}\text { Trial registration } \\
\{2 \mathrm{a} \text { and } 2 \mathrm{~b}\}\end{array}$ & $\begin{array}{l}\text { International Clinical Trials Registry } \\
\text { Platform IRCT20200417047105N1, retrospectively registered at } \\
\text { 19/04/2020. URL: https://irct.ir/trial/47267. }\end{array}$ \\
\hline $\begin{array}{l}\text { Protocol version } \\
\{3\}\end{array}$ & version 2, last updated at Mach 2020. \\
\hline Funding $\{4\}$ & $\begin{array}{l}\text { This work will be financially supported by Mashhad University of } \\
\text { Medical Sciences }\end{array}$ \\
\hline Author details $\{5 \mathrm{a}\}$ & $\begin{array}{l}\text { Malihe Motavasselian, Mohsen Zakerian, } \\
\text { Department of Persian Medicine, School of Persian and } \\
\text { Complementary Medicine, Mashhad University of Medical Sciences, } \\
\text { Mashhad, Iran } \\
\text { Fatemeh Roudi, Reza Rezvani } \\
\text { Department of Nutrition, Faculty of Medicine, Mashhad University of } \\
\text { Medical Sciences, Mashhad, Iran }\end{array}$ \\
\hline $\begin{array}{l}\text { Name and contact } \\
\text { information for } \\
\text { the trial sponsor } \\
\{5 \mathrm{~b}\}\end{array}$ & $\begin{array}{l}\text { This study will be financially supported by the Deputy of Research } \\
\text { and Technology at MUMS (grant \#971727). (Qoreishi Center, } \\
\text { Daneshgah Street, Mashhad, Iran; Email: vcresearch@mums.ac.ir). }\end{array}$ \\
\hline $\begin{array}{l}\text { Role of sponsor } \\
\{5 c\}\end{array}$ & All the study stages will perform under its supervision. \\
\hline
\end{tabular}

\section{Introduction}

\section{Background and rationale $\{6 a\}$}

Resting Energy Expenditure (REE), the required energy for maintaining the basic metabolic activities including keeping the functioning of vital organs such as the lungs, the brain, the kidneys, and the heart and maintaining the body temperature, is considered as the main component of Total Energy Expenditure 
(TEE) [1]. As it is mentioned in previous studies, there are some factors affecting the REE most significantly including body composition (lean body mass), age (independent from the age-related body composition changes), climate, body size and gender [2, 1]. Moreover, thermic effect of food (TEF) is also affected by the composition of the diet $[1,3]$.

Short changes in the energy intake can lead to a number of hormonal and metabolic stimulation alteration in human $[2,4,3]$. Postprandial TEE and TEF are considered as two major objectives in energy balance management [4].

Postprandial hyperglycemia and hypertriglyceridemia are two major independent cardiovascular risk factors which attributed interest for acute phase researches following meal consumption and different dietary exposures as previous studies reported different post prandial levels of triglyceride following consuming different types of fats and also different macronutrient composition of meals [5-8]. Furthermore, as it is reported by Ratliff, et al., Pearce, et al., and Vander, et al. animal protein intake in breakfast can lead to less significant plasma glucose and insulin changes [9-11]. On the other hand, vascular function and arterial stiffness, as independent CVD risk factors, which their damage and intensity are associated with increased risk of CVDs, can be affected by short term dietary exposures while information on postprandial arterial stiffness is limited [12-14].

According to reliable sources of Traditional Persian Medicine (TPM), Temperament as the most basic concept which plays a pivotal role in this medical school, is determined based upon physical and psychological manifestations. It seems that the manifestations of warmth and coldness of temperament and the influential factors such as age, gender, fat and lean body mass, body size, climate, and dietary patterns are in accordance with the REE and TEF [8,15-18]. From the TPM point of view, having more muscle, less age, more body surface (especially in the chest and joints areas), being female and living in a tropical climate indicate a hot temperament [15-19].

On the other hand, all foods are divided into cold/hot-temper and dry/wet-temper and lead to different responses in individuals regarding their temperaments $[8,18,16]$.

Pulse characteristics including volume, speed, strength, intermittency is considered as a main tool distinguishing hot or cold temperament $[8,16,21]$. Notably, vascular function and the probability of cardiovascular events are different in hot and cold-temper subjects as overall cardiovascular functions would be better in a hot-temper individual $[8,17]$.

According to the study of Mohammadi Farsani and his colleagues, there is a probable association between basal metabolic rate and temperament, as BMR in hot-temper individuals would be higher than cold-temper individuals which concur to the rationale of our study $[19,20]$.

In this context, the hypothesis of our study is that different hot/cold temper foods may lead to different metabolic responses and arterial stiffness indices in healthy men with hot and cold TPM-based temperaments. 


\section{Objectives $\{7\}$}

Evaluating and comparison the effects of different TPM-based temperament of isocaloric meals (hot and cold) on metabolic responses (such as energy expenditure components, postprandial glycemia and lipemia) and arterial stiffness indices (pulse wave analysis (PWA) and pulse wave velocity (PWV)) in healthy men with hot and cold TPM-based temperaments.

\section{Trial design $\{8\}$}

This study will be a randomized parallel cross-over clinical trial.

\section{Methods}

\section{Participants, interventions and outcomes}

\section{Study setting $\{9\}$}

The participants of this study will be selected from male students residing in dormitory of Mashhad University of Medical Sciences, Mashhad, Iran, to have a similar life style.

\section{Eligibility criteria $\{10\}$}

Inclusion Criteria:

- Age of 18 to 35 years

- Adults with normal weight (body mass index [BMI] of $18.5-23.5 \mathrm{~kg} / \mathrm{m}^{2}$ )

- Apparently healthy men

- Providing written informed consent

- Willingness to undertake the required fasting periods

- Being TPM-based hot/cold temper

Criteria for not entering the study:

- Being a professional athlete smoking

- Consumption of weight loss or weight gain supplements

- Have significant weight loss over the past 6 months (losing more than $10 \%$ of body weight over the past 6 months)

- Use of certain medications or supplements affecting metabolism (e.g., thyroid drugs, caffeine)

- Positive history of hypertension, diabetes mellitus, hyperlipidemia, CVDs, neurological, and/or neuropsychological disorders

- Consumption of toxic substances; 
Exclusion Criteria:

- Unwillingness to participation continuing

- Inability to partake in the intervention due to dietary preferences /intolerance

- Non-compliance with the terms of calorimetry, and pulse velocimetry

\section{Who will take informed consent? $\{26 \mathrm{a}\}$}

Initially, the objectives of the trial will be clarified by the main administrator to eligible subjects, and written informed consent will be obtained from them at the time of enrolment.

\section{Additional consent provisions for collection and use of participant data and biological specimens \{26b\}}

We do not need any additional consent provision.

\section{Interventions}

\section{Explanation for the choice of comparators $\{6 \mathrm{~b}\}$}

The subjects with eligible criteria will be enrolled to the study.

\section{Intervention description \{11a\}}

The present study will be an acute phase study investigating different effects of two hot/cold TPM based test meals. The Patients with eligible criteria will be enrolled to the study and the hot-cold or cold-hot temper test meals will be administered to each participant according to the sequential randomization plan. Each test day will be implemented during 7 A.M-1 P.M. This period was selected to represent a common interval between breakfast and lunch. The anthropometric measurements will be done at the outset of the fasting state. We will measure the body weight to the nearest 0.1 kilogram, with the subjects in light clothing. The waist circumference will be measured at the midline between the iliac crest and lowest ribs to the nearest 0.5 centimeters. The height of the participants will be measured using a stadiometer in the standing position to the nearest 0.1 centimeter. Finally, we will determine the body composition of the subjects using bioelectrical impedance analysis (AVIS 333). At the intervention day, all participants will have a minimum rest of 15 minutes before the fasting tests and will rest until the end of the tests. All the tests will be performed in the exact same conditions (e.g., quiet area and temperatureregulated room). Firstly, fasting tests including blood sampling, indirect calorimetry (IC), PWV, and PWA will be performed at the fasting state. Secondly, subjects will have 15 minutes to take the complete test meals (under the supervision of researchers). Following the test meals, we will ask all subjects to fast again (refraining from food and drinks (except water)) for another 4 hours. At the next stage, all participants will complete a series of tests (blood sampling, indirect calorimetry (IC), PWV, and PWA) in three times following test meal consumption.

Test meals will be cold-temper meal (including cheese, cucumber, and bread) and hot-temper meal (including steamed camel meat, onion, and bread) and will provide $25 \%$ of daily TEE of participants 
measured by IC in fasting state for each participant. Macronutrient components of both test meals will be similar and include $55 \%$ carbohydrates, $18 \%$ protein and $27 \%$ fat.

Serum samples will be collected to evaluate the markers of metabolic activity. A maximum of three milliliters of blood will be collected from each patient at each of the given times.

For Indirect Calorimetry, we will ask the participants to stay awake and motionless in a supine position, and air samples were collected using a mask (MetaLyzer 3B-R3 device).

The resting energy expenditure and substrate utilization will be measured via indirect calorimetry.

Pulse wave analysis will be done via an ultrasound examination of the carotid artery in supine position.

The evaluation of the brachial blood pressure and pulse wave analysis will perform in supine pine position by the Sphygmocor XCEL device. The augmentation index (Alx), central blood pressure (cBP), and heart rate (HR) will be assessed regarding the guidelines of the manufacturers.

Carotid-radial pulse wave velocity $\left(\mathrm{PWV}_{\mathrm{b}}\right)$ will be determined based upon the applanation tonometry (the sequentially measured electrocardiogram-gated left carotid and radial waveforms; foot-to-foot method for determining the pulse travel time).

\section{Criteria for discontinuing or modifying allocated interventions $\{11 \mathrm{~b}\}$}

Any modification of the protocol that may affect the conduction of the study will be approved by MUMS Ethics Committee prior to implementation. Additionally, it will be announced to the IRCT registration dataset by the researchers.

\section{Strategies to improve adherence to interventions $\{11 \mathrm{c}\}$}

The intervention will be carried out in two different days and as the researchers will accompany all participants in these two days, adherence to the study protocol will be closely monitored. Moreover, we plan to involve the participants in delivering the study dissemination plans.

\section{Relevant concomitant care permitted or prohibited during the trial $\{11 \mathrm{~d}\}$}

Before the intervention, eligibility assessment will be performed. Age, weight, past medical history (in order to be apparently healthy), and willingness to undertake the required fasting periods will be assessed by the research coordinator whom is residing in the dormitory. In the $2^{\text {nd }}$ step, a valid and reliable selfadministered temperament screening questionnaire will be fulfilled by the volunteers [22]; in case of being cold/hot temper by the Mojahedi's temperament assessment questionnaire, the subjects will be visited by three MD, PhDs of Persian medicine. In case of confirmation of the participants' temperament, they will be eligible to enter the study. 
Three days before both test meals all subjects would be asked to have a standardized daily menu plan to be ensured about filled glycogen stores. In addition, participant will be not allowed to engage in severe physical activity within two days prior to the test days. All subjects will arrive at the research site in Imam Reza hospital, Mashhad, Iran by taxi in order to have minimum activity after 12 hours fasting from food, drinks (except water), and caffeine.

\section{Provisions for post-trial care $\{30\}$}

With respect to consuming just two test meals in two different days, no post-trial care is needed.

\section{Outcomes $\{12\}$}

- Metabolic parameters (energy expenditure components including REE, TEF, respiratory exchange ratio (RER), respiratory quotient (RQ), and substrate utilization)

- Non-invasive arterial stiffness markers (Alx, cBP, HR, PWV $\mathrm{b}_{\mathrm{b}}$ )

- Lipemia (including serum triglycerides, free fatty acids, HDL, LDL), glycemia (blood glucose) and insulinemia

\section{Participant timeline $\{13\}$}

Figure 1 shows the study schedule of enrolment, intervention and assessment based in the Standard Protocol Items Recommendations for Interventional Trials (SPIRIT) guidelines.

Figure1. Schedule of enrolment, intervention, and assessments.

\section{Sample size $\{14\}$}

Since we could not find any prior information to base a sample size on, we designed our study as a pilot study. Therefore, we could not assess the attrition rate and there was no necessity to define the sample size.

\section{Recruitment \{15\}}

The participants will be recruited by local advertising with posters at Mashhad University of Medical Sciences. The study will be carried out at the Imam Reza hospital, Mashhad, Iran.

\section{Assignment of interventions: allocation}

\section{Sequence generation $\{16 a\}$}

We generated an online sequential randomization plan, which will be used to develop the allocation sequence and assign the participants to the hot-cold (consumption of hot temper food firstly, and cold temper food secondly) or cold-hot (consumption of cold temper food firstly, and hot temper food secondly) test meals consumption groups by the researchers. 


\section{Concealment mechanism $\{16 \mathrm{~b}\}$}

In this study, there will be just one pre-intervention concealment at the assignment stage of participants to groups.

\section{Implementation \{16c\}}

Generate the allocation sequence, enrol participants, and assign participants to interventions will be done by the main administrative.

\section{Assignment of interventions: Blinding}

\section{Who will be blinded $\{17 \mathrm{a}\}$}

No blinding will be applied for the researchers and participants.

\section{Procedure for unblinding if needed $\{17 \mathrm{~b}\}$}

Not applicable as the researchers and participants will be unblinded about the participants' and the meals tempers during the study.

\section{Data collection and management}

\section{Plans for assessment and collection of outcomes $\{18 \mathrm{a}\}$}

At the intervention day, all participants will have a minimum rest of 15 minutes before the fasting tests and will rest until the end of the tests. All the tests will be performed in the exact same conditions (e.g., quiet area and temperature-regulated room). Firstly, fasting tests including blood sampling, indirect calorimetry (IC), PWV, and PWA will be performed at the fasting state. Secondly, subjects will have 15 minutes to take the complete test meals (under the supervision of researchers). Following the test meals, we will ask all subjects to fast again (refraining from food and drinks (except water)) for another 4 hours. At the next stage, all participants will complete a series of tests (blood sampling, indirect calorimetry (IC), PWV, and PWA) in three times including 0.5-, 2- and 4-hours following test meal consumption.

\section{Plans to promote participant retention and complete follow-up $\{18 \mathrm{~b}\}$}

No follow-up is needed.

Data management \{19\}Data will be recorded in the Excel file and in the paper forms at the same time by the researchers.

\section{Confidentiality $\{27\}$}

The identifiable information of the patients will be stored at the security site, and all the data collection and reports will be identified by a coded ID number. 
Plans for collection, laboratory evaluation and storage of biological specimens for genetic or molecular analysis in this trial/future use $\{33\}$

We will try to collect a minimum two millilitres of blood sample in every round of sampling for hormonal study in future use.

\section{Statistical methods}

\section{Statistical methods for primary and secondary outcomes $\{20 \mathrm{a}\}$}

Descriptive statistics will be used to determine and compare the main characteristics of the subjects between the study groups. The Kolmogorov-Smirnov test will be used to evaluate the normal distribution of the variables. To analyze and inter-group comparison of normally distributed variables, Independentsamples t-test and Chi-square will be used. The Kruskal-Wallis test will be applied for non-parametric data. The P-value of less than 0.05 will be considered as significance level.

\section{Interim analyses $\{21 \mathrm{~b}\}$}

Interim analysis is not considered. In case of any side effect, we will stop the intervention and present the results to the Ethics Committee of Mashhad University of Medical Sciences (MUMS) for further decision.

\section{Methods for additional analyses (e.g. subgroup analyses) \{20b\}}

Any covariates will be controlled by binary logistic regression or ANCOVA test.

Methods in analysis to handle protocol non-adherence and any statistical methods to handle missing data $\{20 \mathrm{c}\}$

As the researchers will accompany all participants in the research site, adherence to the study protocol will be closely monitored.

\section{Plans to give access to the full protocol, participant level-data and statistical code $\{31 \mathrm{c}\}$}

The study results will be submitted for publication as articles to international, peer-reviewed journals. The full protocol and de-identified dataset will be accessible for research objectives.

\section{Oversight and monitoring}

Composition of the coordinating center and trial steering committee $\{5 \mathrm{~d}\}$

The ethical committee and research deputy of Mashhad University of Medical Sciences, supervises all the study stages. It is an academic committee and has no competing interest.

Composition of the data monitoring committee, its role and reporting structure $\{21 \mathrm{a}\}$ 
Adverse event reporting and harms $\{22\}$

In case of any side effect, we will stop the intervention and present the results to the Ethics Committee of Mashhad University of Medical Sciences (MUMS) for further decision.

\section{Frequency and plans for auditing trial conduct $\{23\}$}

Auditing trial conduct is not considered

\section{Plans for communicating important protocol amendments to relevant parties (e.g. trial participants, ethical committees) $\{25\}$}

Any modification to protocol which may impact on the conduct of the study, will be approved by ethical committee of MUMS prior to implementation.

\section{Dissemination plans $\{31 \mathrm{a}\}$}

The primary RCT results will be submitted for publication to an international, peer-reviewed journal. Authorship eligibility will be based on the recommendations from the International Committee of Medical Journal Editors (ICMJE).

\section{Discussion}

In TPM school, paying attention to nutrition and its modification has been superior to prescribing medication including herbal/ chemical drugs and various interventional procedures as it is considered as the first step of life style modification in order to disease prevention and treatment $[15,16,18]$. In this medicine, foods are considered to have their own nature, which requires people with different temperaments to consume foods that are in accordance with their temperaments to maintain their health and cure their diseases $[15,16,18]$. According to our search, there is a lack of evidence in investigating the possible association between temperament, food intake, and metabolism.

As it is reported by Mohammadi Farsani G, et al., cold temper subjects had a non-significant lower BMR; while they had significantly lower heart rate, and systolic and diastolic blood pressure [20]. But they mentioned that they would have an interventional phase investigating the acute phase effect of thyme/ sumac consumption as cold and hot temper agents on metabolic responses and sympathetic nervous system activity of the body that its results are not published yet [23]. As it is aforementioned, modification of the routine diet is superior to herbal/chemical drug usage in TPM school. Furthermore, the heterogenicity of the participants' ages (18-40 years) in this study may lead to different inter- and intraindividuals' metabolic and sympathetic responses.

The current research project aim is to investigate the effects of the single ingestion of TPM-based hot/cold test meals on the vascular reactivity and metabolic responses of healthy adults. To the best of 
our knowledge, no previous study assessed the acute postprandial effects of standard isocaloric meals with various TPM-based temperaments on metabolic and vascular parameters.

Given that the prevalence of CVDs is increasing rapidly and as there is close correlation between CVDs and diets, lipemia, and glycemia after meals; investigating the effects of meal temperaments on vascular functions and metabolic responses in hot/cold temper subjects would be of great interest. It is estimated that the main challenge in this research project will be the participants' compliance.

Regarding the design of our study, we will be ensured about the balance in the confounding variables in both study groups.

This experiment could be incorporated into the design of novel TPM and modern nutrition based dietary interventions for adapting the type of various people to the type of foods in order to improve metabolic responses and vascular reactivity.

\section{Trial Status}

During the submission of this document, the sampling of the patients started. The recruitment was started by March 2020 and it is estimated to be completed by November 2020. Recruitment was ongoing at the time of submission.

\section{Abbreviations}

Traditional Persian Medicine (TPM) Total Energy Expenditure (REE) Resting Energy Expenditure (REE) Thermic Effect of Food (TEF) Body Mass Index (BMI) Indirect Calorimetry (IC) Augmentation index (Alx) Central Blood Pressure (cBP) Heart Rate (HR) Carotid-radial pulse wave velocity (PWVb) Pulse Wave Analysis (PWA) Pulse Wave Velocity (PWV) Respiratory Exchange Ratio (RER) Respiratory Quotient (RQ)

\section{Declarations}

\section{Acknowledgements}

The authors would like to thank all the students who will participate and all the medical staff at Nutrition and Persian Medicine departments for assisting us in this study.

\section{Authors' contributions $\{31 \mathrm{~b}\}$}

$M Z, F R, R R$ and $M M$ were involved in the study design. $M Z$, and FR will contribute data collection, and data analysis. MZ, FR, RR, and MM contributed to development of the protocol. All the authors read and approved the final manuscript.

\section{Funding $\{4\}$}


This study will be financially supported by the Deputy of Research and Technology at MUMS (grant \#971727). (Qoreishi Center, Daneshgah Street, Mashhad, Iran; Email: vcresearch@mums.ac.ir). All study stages such as design of the study and data collection, analysis, interpretation of data and in writing the manuscript will be undertaken under its supervision.

\section{Availability of data and materials $\{29\}$}

The de-identifiable data will be available only to the researchers. The data could be obtained via email by Dr. Malihe Motavasselian (Email: MotavasselianM@mums.ac.ir). Other researchers in academic institutions could also send their request via email, and they will receive the data after consultation and approval of the research team.

\section{Ethics approval and consent to participate $\{24\}$}

The protocol of this study was approved by the Ethics committee of Mashhad University of Medical Sciences (Ethical Code: IR.MUMS.REC.1398.014).

All the patients will provide written informed consent.

\section{Consent for publication $\{32\}$}

Although in this study there are no individual details, images or videos but confidentiality principles will be fully respected.

\section{Competing interests $\{28\}$}

None declared.

\section{Author details}

Malihe Motavasselian, and Mohsen Zakerian,

Department of Persian Medicine, School of Persian and Complementary Medicine, Mashhad University of Medical Sciences, Mashhad, Iran

Fatemeh Roudi, and Reza Rezvani

Department of Nutrition, Faculty of Medicine, Mashhad University of Medical Sciences, Mashhad, Iran

\section{References}

1. Mahan LK, Raymond JL. Krause's food \& the nutrition care process. 2017.

2. Ndahimana D, Kim E-K. Measurement Methods for Physical Activity and Energy Expenditure: a Review. Clin Nutr Res. 2017;6(2):68-80. doi:10.7762/cnr.2017.6.2.68. 
3. Arciero PJ, Ormsbee MJ, Gentile CL, Nindl BC, Brestoff JR, Ruby M. Increased protein intake and meal frequency reduces abdominal fat during energy balance and energy deficit. Obesity (Silver Spring, Md). 2013;21(7):1357-66. doi:10.1002/oby.20296.

4. Morgan JB, York DA, Wasilewska A, Portman J. A study of the thermic responses to a meal and to a sympathomimetic drug (ephedrine) in relation to energy balance in man. The British journal of nutrition. 1982;47(1):21-32. doi:10.1079/bjn19820005.

5. Cavalot F, Petrelli A, Traversa M, Bonomo K, Fiora E, Conti M et al. Postprandial blood glucose is a stronger predictor of cardiovascular events than fasting blood glucose in type 2 diabetes mellitus, particularly in women: lessons from the San Luigi Gonzaga Diabetes Study. The Journal of clinical endocrinology and metabolism. 2006;91(3):813-9. doi:10.1210/jc.2005-1005.

6. Nordestgaard BG, Benn M, Schnohr P, Tybjærg-Hansen A. Nonfasting Triglycerides and Risk of Myocardial Infarction, Ischemic Heart Disease, and Death in Men and Women. JAMA. 2007;298(3):299-308. doi:10.1001/jama.298.3.299.

7. Bansal S, Buring JE, Rifai N, Mora S, Sacks FM, Ridker PM. Fasting compared with nonfasting triglycerides and risk of cardiovascular events in women. Jama. 2007;298(3):309-16. doi:10.1001/jama.298.3.309.

8. Peairs AD, Rankin JW, Lee YW. Effects of acute ingestion of different fats on oxidative stress and inflammation in overweight and obese adults. Nutrition Journal. 2011;10(1):122. doi:10.1186/14752891-10-122.

9. Ratliff J, Leite JO, de Ogburn R, Puglisi MJ, VanHeest J, Fernandez ML. Consuming eggs for breakfast influences plasma glucose and ghrelin, while reducing energy intake during the next 24 hours in adult men. Nutrition research (New York, NY). 2010;30(2):96-103.

doi:10.1016/j.nutres.2010.01.002.

10. Pearce KL, Clifton PM, Noakes M. Egg consumption as part of an energy-restricted high-protein diet improves blood lipid and blood glucose profiles in individuals with type 2 diabetes. The British journal of nutrition. 2011;105(4):584-92. doi:10.1017/s0007114510003983.

11. Vander Wal JS, Marth JM, Khosla P, Jen KL, Dhurandhar NV. Short-term effect of eggs on satiety in overweight and obese subjects. Journal of the American College of Nutrition. 2005;24(6):510-5. doi:10.1080/07315724.2005.10719497.

12. Deanfield JE, Halcox JP, Rabelink TJ. Endothelial function and dysfunction: testing and clinical relevance. Circulation. 2007;115(10):1285-95. doi:10.1161/circulationaha.106.652859.

13. Wilkinson IB, McEniery CM. Arterial stiffness, endothelial function and novel pharmacological approaches. Clinical and experimental pharmacology \& physiology. 2004;31(11):795-9. doi:10.1111/j.1440-1681.2004.04074.x.

14. Ahuja KDK, Robertson IK, Ball MJ. Acute effects of food on postprandial blood pressure and measures of arterial stiffness in healthy humans. The American Journal of Clinical Nutrition. 2009;90(2):298-303. doi:10.3945/ajcn.2009.27771. 
15. Avicenna. The Canon of medicine. Translated by Sharafkandi AR. Tehran: Soroush press; 2008 [in Persian].

16. Aghili SMH. The Principal's of Traditional Iranian Medicine (Kholassat Al-Hekmah). Nazem E, editor. Qom, Iran: Esmaeilian; 2006. [in Persian].

17. Laila Shirbeigi, Azadeh Zarei, Ayeh Naghizadeh, Vaghasloo MA. The Concept of Temperaments in Traditional Persian Medicine. Trad Integr Med. 2017;2(3):143-56.

18. Ahvazi AEA. The Complete Art of Medicine (Kamil al-Sinaat al-Tibbiyya). Edited by Institute of Natural Medicine Reivivition. Qom: Jallaleddin; 2008. [in Arabic].

19. Mohammadi Farsani G, Naseri M, Movahed M, Dorosti Motlagh A. The Association between Basal Metabolic Rate and Temperament in Iranian Traditional Medicine point of view. Journal of Islamic and Iranian Traditional Medicine. 2017;8(1):29-34.

20. Mohammadi Farsani G, Naseri M, Hosseini S, Saboor-Yaraghi AA, Kamalinejad M, Mohammadi Farsani T, et al. The Evaluation of basic and neurohormonal parameters in hot or cold temperament person proposed in Iranian Traditional Medicine: an observational study. Journal of Contemporary Medical Sciences. 2020;6(4).

21. Nazem Jahan M. Exir-e A'zam. Tehran: Iran University of Medical Sciences, Institute of Medicine History, Islamic and Alternative Medicine 2008.

22. Mojahedi M, Naseri M, Majdzadeh R, Keshavarz M, Ebadini M, Nazem E et al. Reliability and Validity Assessment of Mizaj Questionnaire: A Novel Self-report Scale in Iranian Traditional Medicine. Iran Red Crescent Med J. 2014;16(3):e15924-e. doi:10.5812/ircmj.15924.

23. Farsani GM, Movahhed M, Motlagh AD, Hosseini S, Yunesian M, Farsani TM, et al. Is the Iranian Traditional Medicine warm and cold temperament related to Basal Metabolic Rate and activity of the sympathetic-parasympathetic system? Study protocol. Journal of Diabetes \& Metabolic Disorders. 2014;13(1):74.

\section{Table}

Table 1. Schedule of enrolment, intervention, and assessments. 


\begin{tabular}{|c|c|c|c|c|c|c|}
\hline TIMEPOINT & $\begin{array}{c}\text { Before } \\
\text { intervention }\end{array}$ & baseline & $\begin{array}{c}\text { Meal } \\
\text { consumption } \\
\text { (0:00) }\end{array}$ & $0: 30$ & $2: 00$ & $4: 00$ \\
\hline Eligibility screen & $X$ & & & & & \\
\hline Informed consent & $X$ & $X$ & & & & \\
\hline Obtaining 3-day food recall & & $X$ & & & & \\
\hline $\begin{array}{c}\text { Determining body } \\
\text { composition }\end{array}$ & & $X$ & & $X$ & $X$ & $X$ \\
\hline $\begin{array}{c}\text { Determining physical } \\
\text { activity Level }\end{array}$ & & $X$ & & $X$ & $X$ & $X$ \\
\hline Anthropometric assessment & & $X$ & & $X$ & $X$ & $X$ \\
\hline Indirect calorimetry & & $X$ & & & \\
\hline PWA \& PWV & & $X$ & & & \\
\hline Blood sampling & & & & & & \\
\hline
\end{tabular}

